\title{
Update and evaluation of decay data for spent nuclear fuel analyses
}

\author{
Teodosi Simeonov ${ }^{1, a}$ and Charles Wemple ${ }^{2}$ \\ 1 Studsvik Scandpower Inc., Waltham, Massachusetts, USA \\ 2 Studsvik Scandpower Inc., Idaho Falls, Idaho, USA
}

\begin{abstract}
Studsvik's approach to spent nuclear fuel analyses combines isotopic concentrations and multigroup cross-sections, calculated by the CASMO5 or HELIOS2 lattice transport codes, with core irradiation history data from the SIMULATE5 reactor core simulator and tabulated isotopic decay data. These data sources are used and processed by the code SNF to predict spent nuclear fuel characteristics. Recent advances in the generation procedure for the SNF decay data are presented. The SNF decay data includes basic data, such as decay constants, atomic masses and nuclide transmutation chains; radiation emission spectra for photons from radioactive decay, alpha-n reactions, bremsstrahlung, and spontaneous fission, electrons and alpha particles from radioactive decay, and neutrons from radioactive decay, spontaneous fission, and alpha-n reactions; decay heat production; and electro-atomic interaction data for bremsstrahlung production. These data are compiled from fundamental (ENDF, ENSDF, TENDL) and processed (ESTAR) sources for nearly 3700 nuclides. A rigorous evaluation procedure of internal consistency checks and comparisons to measurements and benchmarks, and code-to-code verifications is performed at the individual isotope level and using integral characteristics on a fuel assembly level (e.g., decay heat, radioactivity, neutron and gamma sources). Significant challenges are presented by the scope and complexity of the data processing, a dearth of relevant detailed measurements, and reliance on theoretical models for some data.
\end{abstract}

\section{Introduction}

Historically, several decay parameters needed for spent nuclear fuel analyses have been derived from data sources outside the evaluated nuclear data files. Recent advances in ENDF [1], ENSDF [2] and TENDL [3] created a basis for a straight forward procedure for compilation and evaluation of a complete set of decay data. Such an approach has the advantage of solely using publically available, widely used, well-maintained, well-documented, and regularly updatable databases as the foundation. This approach was adopted by Studsvik in the update of the decay data library of its code for spent fuel analyses, SNF [4].

The first objective in the process was to create a procedure to compile and evaluate a complete set of all available decay data for all available isotopes. The status of that first step is outlined in this paper along with a few issues encountered during the data evaluation and internal consistency checks.

\section{Decay data sources and evaluation}

\subsection{Basic decay data}

Data for 3691 isotopes were processed from the ENDF/BVIIR1 [1] decay sub-library. The basic decay data set is designed to provide sufficient, and as complete as possible, information to build the transformation matrix describing the radioactive decay.

\footnotetext{
a e-mail: teodosi.simeonov@studsvik.com
}

The basic decay data saved per isotope in the decay file are: the half-life with uncertainty; atomic mass; decay modes and branching ratios with uncertainty; decay mode Q-values with uncertainty; and the daughter isotopes.

The evaluation is merely a book-keeping exercise, involving cross checks with other nuclear data evaluations and/or earlier revisions. All encountered differences were deemed either improvements in the evaluated data or within the given uncertainty range.

\subsection{Decay spectra}

The decay spectrum specifies the particular particle emission accompanying each decay mode. These are weighted sums from all decay modes emitting the particle.

\subsubsection{Discrete spectra}

The discrete spectra for alpha, gamma and $\mathrm{x}$-ray are provided in the decay file along with the energy line uncertainty.

\subsubsection{Spontaneous fission spectra}

For neutrons from spontaneous fission (SF) of actinides (except for ${ }^{252} \mathrm{Cf}$ ), the emitted spectrum is a MaxwellBoltzmann distribution at the temperature of the average neutron energy, normalized to the average number of neutrons emitted from spontaneous fission for that nuclide.

When SF neutron emission data were not available from the ENDF decay sub-library, the average number of emitted neutrons and spectrum temperature were obtained 
from [5]. For all other histogram and discrete spectra, the spectrum is normalized to the total emission per decay [6], so that particle emission rates can be calculated directly from the decay constant, branching ratio, and spectrum.

\subsubsection{Beta spectra}

For nuclides with discrete spectra provided for beta and/or positron decay, the energy provided in the ENDF decay sub-library is the total decay energy. This must be converted to a continuous spectrum for the emitted electron or positron. For beta decay, the maximum emitted electron energy is given by the total decay energy and the spectrum can be calculated using Fermi's theory [7]:

$$
N(Z, E) \propto F(Z, E) * K_{j}(E) * p * E *\left(E_{0}-E\right)^{2}
$$

where $\mathrm{Z}$ is the atomic number of the daughter nucleus, $\mathrm{E}$ is the electron total (kinetic + rest mass) energy, $\mathrm{F}$ is the electron density ratio (Fermi function), $\mathrm{K}_{\mathrm{j}}$ is a shape factor for the specific decay type (allowed, first forbidden, etc.), $\mathrm{p}$ is the electron momentum, and $\mathrm{E}_{0}$ is the maximum electron energy for the decay.

A simple and fairly accurate analytic approximation for the Fermi function was derived by Schenter and Vogel [8]:

$$
F(Z, E)=\frac{E}{p} \exp \left[\alpha(Z)+\beta(Z)(W-1)^{0.5}\right]
$$

where $\mathrm{W}$ is the total electron energy expressed in electron rest-mass units, and $\alpha$ and $\beta$ are fitted parameters.

The shape function for the allowed $(\mathrm{j}=0)$, firstforbidden $(j=1)$ and second-forbidden $(j=2)$ transitions are given by [9]:

$$
\begin{aligned}
& K_{0}(E)=1 \\
& K_{1}(E)=\left(E^{2}-m_{e} c^{2}\right)+\left(E_{0}-E\right)^{2} \\
& K_{2}(E)=\left(E^{2}-m_{e} c^{2}\right)^{2}+\left(E_{0}-E\right)^{4}+\frac{10}{3}\left(E^{2}-m_{e} c^{2}\right)\left(E_{0}-E\right)^{2}
\end{aligned}
$$

This allows the beta energy spectrum to be calculated by numerical integration over the global energy grid, with the resulting spectrum normalized to the total beta emission.

To calculate the positron spectrum from electron capture/positron decay, two adjustments must be made to the beta spectrum formulation. First, the maximum positron energy, $E_{0}$, is given by subtracting twice the electron rest mass energy from the maximum decay energy. This is necessary to permit creation of the positron; if this is not energetically possible, the decay proceeds by electron capture. Second, the spectrum must be phaseshifted by the ratio of the positron (negative $\eta$ ) and electron (positive $\eta$ ) Fermi functions. After application of a property of the gamma function [10]:

$$
\Gamma(a+i b)=\frac{\Gamma(1+a)}{a} \prod_{n=0}^{\infty}\left[1+\frac{b^{2}}{(a+n)^{2}}\right]^{1 / 2}
$$

and cancellation of identical terms, the ratio is reduced to:

$$
r_{F}=e^{-2 \pi \eta}
$$

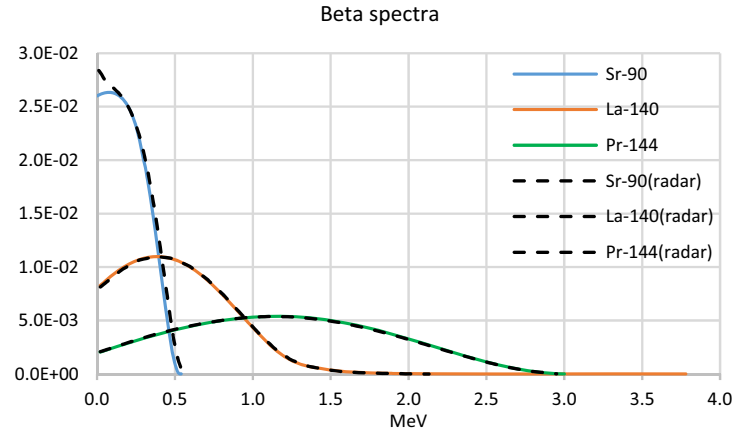

Figure 1. Beta decay spectra evaluation.

This is applied to the Fermi function expression of Eq. (2) during the numerical integration and the resulting spectrum is normalized to the total positron emission.

The importance of accurate evaluation of the beta spectra is significant, because they affect directly the evaluation of bremsstrahlung photons and spectra. An available source of beta spectra is the RADAR data base [11]. Comparisons of sample spectra for several important beta emitters is shown in Fig. 1.

\subsection{Data for $(\alpha, n)$ reactions}

The $(\alpha, n)$ reaction data file for 43 isotopes were processed from TENDL-2014 by NJOY-2012 code, update 40 [12] with the alpha particle stopping powers for the elements including compounds and mixtures derived from ASTAR [13].

Recent releases of TENDL [3] have included $\alpha$ interaction cross section data derived from optical model calculations, including neutron and photon emission spectrum data.

The TENDL evaluations for the oxygen isotopes ${ }^{17} \mathrm{O}$ and ${ }^{18} \mathrm{O}$ do not include the resonance structure below about $5 \mathrm{MeV}$. Historically, $(\alpha, \mathrm{n})$ cross section data for the oxygen isotopes have been taken directly or derived from Perry and Wilson [14], as there have been limited sources of evaluated $\alpha$-interaction cross section data. These data have always represented total neutron production, not production matrices, and so have not been able to provide emitted neutron spectra. The approach used to derive the ${ }^{17} \mathrm{O}$ and ${ }^{18} \mathrm{O}(\alpha, \mathrm{n})$ cross sections for SNF was to rescale the lower-energy data of Perry and Wilson to match the TENDL data at the upper energy of the resonance range $\left(5.404 \mathrm{MeV}\right.$ for ${ }^{17} \mathrm{O}$ and $5.18 \mathrm{MeV}$ for $\left.{ }^{18} \mathrm{O}\right)$; the rescaling increased the cross sections below these energies by $\sim 25 \%$ and $\sim 4 \%$, respectively. This allowed the cross section representation to be extended to the upper limit of the SNF $\alpha$-decay data $(\sim 13 \mathrm{MeV})$, or to higher energies in the future, if necessary. For all other isotopes, the TENDL data were used exclusively, as they agreed well with past measurements [15]. The effect of the adjustment on the total neutron yields is illustrated in Fig. 2, with the related neutron spectra in Fig. 3. The adjusted total neutron yield for UO2 is shown in Fig. 4.

\subsection{Bremsstrahlung data}

The external bremsstrahlung cross sections and production kernels were processed from the ENDF/B-VIIR1 [1] 


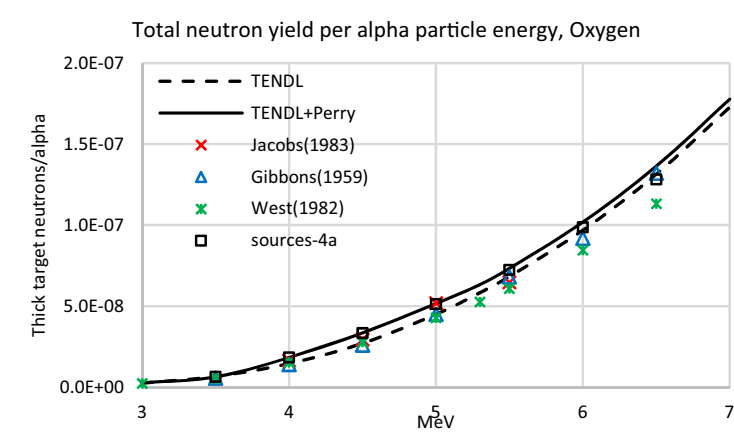

Figure 2. Oxygen, $(\alpha, \mathrm{n})$ total neutron yields. Perry [14], Jacobs [16], Gibbons [17], West [18], SOURCES-4A [19].

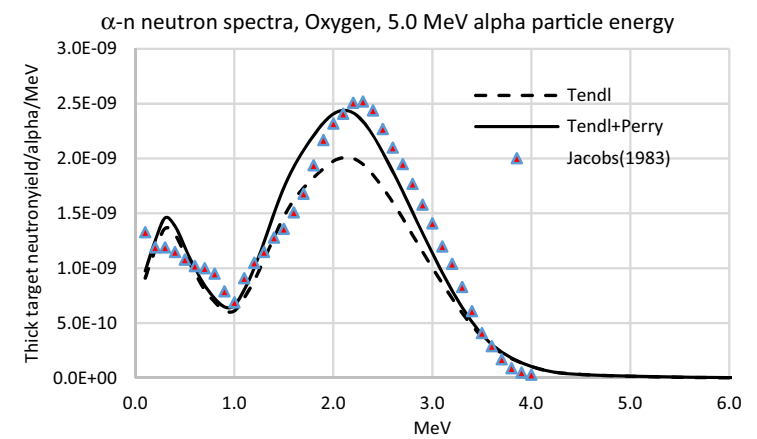

Figure 3. Oxygen $(\alpha, n)$ neutron spectra. Perry [14], Jacobs [16].

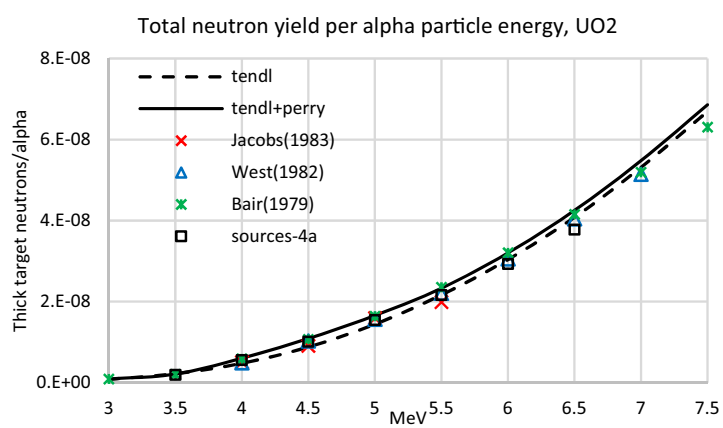

Figure 4. UO2 $(\alpha, \mathrm{n})$ total neutron yields. Perry [14], Jacobs [16], West [18], Bair [15], SOURCES-4A [19].

electron sub-library, while the electron stopping power data were calculated with the ESTAR application [13].

The electron interaction data were extracted from the elemental ENDF electron cross section library, which provides data for the first 100 elements $(\mathrm{H}-\mathrm{Fm})$ at incident electron energies from $10 \mathrm{eV}$ to $100 \mathrm{GeV}$. The cross section data are provided as a linearly interpolated piecewise function in incident electron energy [6]. The scattering matrices are provided as a two-dimensional interpolated function of incident electron energy and bremsstrahlung photon energy, with linear interpolation in each dimension, and the photon spectrum at any energy normalized to unit integral [6]. Strict linear interpolation between spectra at different incident electron energies can produce non-physical artefacts (i.e., up-scatter) in the interpolated spectra. This was avoided by using unit-base interpolation, where the maximum interpolated spectrum energy is limited by the incident electron energy, with the standard normalization applied.

The ESTAR [13] system provides stopping power data for electrons incident on more than 250 materials,
Table 1. Bremsstrahlung radiation yield relative to ESTAR.

\begin{tabular}{l|lll}
$\mathbf{M e V}$ & $\mathbf{O}$ & $\mathbf{U}$ & $\mathbf{U O 2}$ \\
\hline $\mathbf{0 . 2}$ & 1.730 & 1.705 & 1.113 \\
$\mathbf{0 . 5}$ & 1.560 & 1.195 & 0.921 \\
$\mathbf{1 . 0}$ & 1.808 & 1.430 & 1.117 \\
$\mathbf{1 . 5}$ & 1.611 & 1.791 & 1.174 \\
$\mathbf{2 . 0}$ & 1.414 & 1.711 & 1.090 \\
$\mathbf{2 . 5}$ & 1.351 & 1.532 & 1.029 \\
$\mathbf{3 . 0}$ & 1.357 & 1.447 & 1.021 \\
$\mathbf{3 . 5}$ & 1.394 & 1.419 & 1.042 \\
$\mathbf{4 . 0}$ & 1.447 & 1.426 & 1.080
\end{tabular}

including the first 98 elements $(\mathrm{H}-\mathrm{Cf})$, at electron energies from $10 \mathrm{keV}$ to $100 \mathrm{GeV}$. The collision and radiative (bremsstrahlung) components are calculated, along with the total stopping power. Two approximations were necessary to extend the data to the full range needed for SNF [4]. Stopping powers for Cf were also used for Es and Fm to provide data for the full range of elements. For all elements, the stopping power data were extrapolated below $10 \mathrm{keV}$ to provide stopping powers down to $0.1 \mathrm{eV}$, using log-log energy dependence. This method is consistent with the energy dependence of the collision stopping power, which dominates the total stopping power at low energies [13].

The evaluation of bremsstrahlung photon spectra presented a serious challenge, because no published measured data at beta energies below $5 \mathrm{MeV}$ was found. Comparisons to bremsstrahlung data calculated by EDISTR [20] and available as part of the SCALE/ORIGEN [21] photon libraries show a general under prediction at energies below $0.5 \mathrm{MeV}$ with an over prediction observed above $1.0 \mathrm{MeV}$. Consistency checks with the radiation yields from ESTAR suggested over prediction at the lower range, as well, as shown in Table 1. This apparent contradiction has not yet been fully explained.

\subsection{SF fission yields}

The SF independent and cumulative yields for each SF isotope were compiled from ENDF/B-VIIR1 and included, for the first time, in the data file.

The independent yields of all fission product isotopes included in the data file for each SF isotope should sum to 2.0, within the uncertainty limits of the yield data. For each SF isotope presently included in the data file, the independent yield sum is within $0.0001 \%$ of 2.0 .

\subsection{Recoverable energy}

The recoverable energies provided in ENDF/B-VIIR1 [1] are largely updated and more complete compared to the earlier versions of the ENDF decay libraries.

The average energy available as decay heat provides the energy available from an average decay (weighted sum over all decay modes; neutrinos excluded) that may be applied to the decay heat calculation. The three components provided in the data file: are the sum over all light particles $\left(\beta^{-}, \beta^{+}\right.$, conversion and Auger electrons, etc.); the sum over all electromagnetic radiation types (gamma, x-ray, internal Bremsstrahlung, annihilation photons, etc.); and the sum over all heavy particles (proton, neutron, $\alpha$, etc.). The uncertainties, in $\mathrm{eV}$, are also provided for each component. If spontaneous fission (SF) is defined 


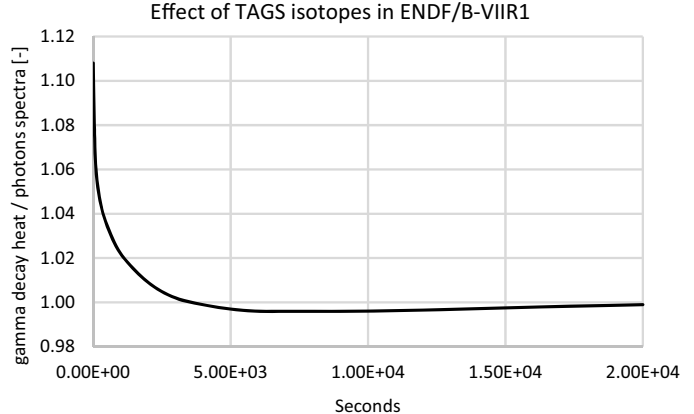

Figure 5. Average EEM relative photon spectra energy.

as a decay path, an extra component of the decay heat is calculated as the SF Q-value weighted by the SF branching ratio.

For some isotopes, the ENDF decay data does not provide a Q-value for spontaneous fission. In this case, a value is provided, or one is calculated from the neutroninduced fission energy components. The recoverable energy is then approximated as:

$$
E_{S F}=E_{f f}+E_{n p}+0.5 *\left(E_{g p}+E_{g d}+E_{\beta}\right)
$$

where the subscripts refer to the fission fragments $(f f)$, prompt neutrons $(n p)$, prompt gammas $(g p)$, delayed gam$\operatorname{mas}(g d)$, and betas $(\beta)$, respectively, released during spontaneous fission. As the range of SF recoverable Qvalues for the actinides is $\sim 170-220 \mathrm{MeV}$ with typical uncertainties of $1-2 \%$, an uncertainty of $10 \%$ is conservatively applied to values thus computed.

As for the energy from electromagnetic radiation, a discrepancy between the evaluated mean energy and the energy computed by integration of calculated photons spectra for decay times up to $2 \times 10^{4} \mathrm{~s}$ was observed. The explanation was found in ENDF corrections related to the so called "pandemonium" effect, in which the inclusion of high efficiency data from TAGS (Total Absorption Gamma Spectrometry) in the evaluation of the mean EM energy, created inconsistencies with the individual photon energies [22]. There were 48 isotopes with a deviation greater than $0.5 \%$. Four of these $48-{ }^{145} \mathrm{Ba},{ }^{142} \mathrm{La},{ }^{155} \mathrm{Nd}$, and ${ }^{155} \mathrm{Pm}$ - produced integrated photon spectra decay heat greater than the mean energy. Those four were responsible for a $0.3 \%$ drop in the total effect of TAGS isotopes, as shown on Fig. 5.

\section{Summary and future work}

A procedure for decay data updates, based nearly entirely on available evaluated nuclear data files, was established and data consistency was found to be generally satisfactory. The few remaining open questions, e.g., lower energy bremsstrahlung photons, will be pursued by further checks of the processing methodology, data sources, and validation against relevant measurements.

The decay data file will be applied to Studsvik's system for spent fuel analyses and released for production after passing through the SNF code validation suite. Initial tests have already indicated consistency for its standard application to LWR assemblies. With the lattice codes (CASMO5 and HELIOS2) and the nodal core simulator (SIMULATE5), this will complete an integrated suite of codes for fuel cycle analysis based on the most recent evaluated nuclear databases.

\section{References}

[1] M.B. Chadwick, et al., Nucl. Data Sheets 112(12), 2887 (2011)

[2] J.K. Tuli, "Evaluated Nuclear Structure Data File", BNL-NCS-51655-01/02-Rev, BNL (2001)

[3] A.J. Koning and D. Rochman, Nucl. Data Sheets 113(12), 2841 (2012). Database available from ftp://ftp.nrg.eu/pub/www/talys/tendl2014/ tendl2014.html

[4] S. Børresen, "Spent Fuel Analyses based on In-Core Fuel Management Calculations", PHYSOR 2004, Chicago, Illinois, April 25-29 (2004)

[5] H. Matsunobu, et al., "Data book for calculating Neutron Yields from $(\alpha, n)$ Reaction and Spontaneous Fission", JAERI 1324 (1992)

[6] M.Herman, A.Trkov (ed.), "ENDF-6 Formats Manual”, ENDF-102(BNL-90365-2009 Rev1), §8.4.2, BNL (2010)

[7] E. Fermi, Zeitschrift für Physik 88(3-4), 161 (1934)

[8] G.K. Schenter and P. Vogel, Nucl. Sci. Eng. 83(3), 393 (1983)

[9] M.G. Stamatelatos and T.R. England, Nucl. Sci. Eng. 63(2), 204 (1977)

[10] G. Arfken, Mathematical Methods for Physicists, 3rd ed., Academic Press (1985), p. 548

[11] M.G. Stabin, Health Phys 83(4), 471 (2002)

[12] A.C. Kahler (ed.), "The NJOY Version 2012”, LAUR-12-27079, (2012). (Updated for NJOY2012.40, 2014)

[13] M.J. Berger, et al., "ESTAR, PSTAR, and ASTAR", NIST, (2015). Online at http://physics.nist.gov/Star

[14] R.T. Perry and W.B. Wilson, "Neutron Production from $(\alpha, \mathrm{n})$ Reactions and Spontaneous Fission in ThO2, UO2, and (U,Pu)O2 Fuels", LA-8869-MS, LANL (1981)

[15] J.K. Bair and J. Gomez del Campo, Nucl. Sci. Eng. 71, 18 (1979)

[16] G.J.H. Jacobs and H. Liskien, Ann. Nucl. Ener. 10, $541(1983)$

[17] J.H. Gibbons, R.L. Masklin, Phys. Rev. 114, 571 (1959)

[18] D. West and A.C. Sherwood, Ann. Nucl. Ener. 9, 551 (1982)

[19] W.B. Wilson at al. "SOURCES-4C: A code for calculation ( $\mathrm{a}, \mathrm{n})$, spontaneous fission and delayed neutron sources and spectra.", LA-UR-02-1839, LANL (2002)

[20] L.T. Dillman, "EDISTR - A computer program to obtain a nuclear decay data base for radiation dosimetry", ORNL/TM6689 (1980)

[21] I.C. Gauld, D. Wiarda, "ORIGEN-S Data Libraries", ORNL/TM-2005/39 v6.1, §M6 (2011)

[22] A. Algora, et al., Phys. Rev. Lett. 105, 202501 (2010) 